\title{
Reduced genetic influence on childhood obesity in small for gestational age children
}

Dug Yeo Han ${ }^{1,3^{*}}$, Rinki Murphy ${ }^{4}$, Angharad R Morgan ${ }^{1,3}$, Wen Jiun Lam, ${ }^{1,3}$ John M D Thompson², Clare R Wall', Karen E Waldie ${ }^{2}$, Edwin A Mitchell ${ }^{2^{*}}$ and Lynnette R Ferguson ${ }^{1,3^{*}}$

\begin{abstract}
Background: Children born small-for-gestational-age (SGA) are at increased risk of developing obesity and metabolic diseases later in life, a risk which is magnified if followed by accelerated postnatal growth. We investigated whether common gene variants associated with adult obesity were associated with increased postnatal growth, as measured by BMI z-score, in children born SGA and appropriate for gestational age (AGA) in the Auckland Birthweight Collaborative.

Methods: A total of 37 candidate SNPs were genotyped on 547 European children (228 SGA and 319 AGA). Repeated measures of BMI (z-score) were used for assessing obesity status, and results were corrected for multiple testing using the false discovery rate.

Results: SGA children had a lower BMI z-score than non-SGA children at assessment age 3.5, 7 and 11 years. We confirmed 27 variants within 14 obesity risk genes to be individually associated with increasing early childhood BMI, predominantly in those born AGA.

Conclusions: Genetic risk variants are less important in influencing early childhood BMI in those born SGA than in those born AGA, suggesting that non-genetic or environmental factors may be more important in influencing childhood BMI in those born SGA.
\end{abstract}

Keywords: BMI, Childhood obesity, AGA children, SGA children

\section{Background}

Worldwide more than 30 million infants per year are born small for gestational age (SGA) defined by birth weight below the 10th percentile, according to gestational age [1]. These infants are at increased risk of mortality and morbidity in early life as well as greater later risk of developing obesity, dyslipidemia, hypertension and diabetes.

It has been suggested that growth restriction in the womb resulting from poor maternal nutrition, can program the individual's physiology and metabolism predisposing them to long-term susceptibility to metabolic

\footnotetext{
* Correspondence: dy.han@auckland.ac.nz; e.mitchell@auckland.ac.nz; I. ferguson@auckland.ac.nz

'Discipline of Nutrition, FM\&HS, The University of Auckland, Auckland, New Zealand

${ }^{2}$ Department of Paediatrics, FM\&HS, The University of Auckland, Auckland, New Zealand

${ }^{3}$ Nutrigenomics New Zealand, Auckland, New Zealand

Full list of author information is available at the end of the article
}

diseases [2,3]. Alternative hypotheses explaining the link between low birthweight and higher risk of adult metabolic diseases include the foetal insulin hypothesis [4] that suggests common genetic variants which influence insulin secretion or action may both reduce foetal growth (as insulin is a key foetal growth factor) and increase the risk of type 2 diabetes in later life.

Candidate gene and genome wide association studies have led to the discovery of over 50 gene variants known to be associated with adult obesity $[5,6]$. Two recent studies involving the Avon Longitudinal Study of Parents and Children [7], and the 1946 British Birth Cohort study [8], have found that a genetic predisposition score of 8 and 11 adult obesity susceptibility alleles were associated with greater infancy and childhood weight, height and BMI gain. The obesity risk allele score showed a weaker association with BMI than with weight until 3.5 years, due to concurrent gains in both length and weight during infancy. While combining established 
genetic variants for BMI into a risk-allele score maximised statistical power, this approach masked possible heterogeneity in effects of individual variants.

The detrimental effect of poor fetal growth on long-term metabolic health is observed to be magnified if followed by accelerated postnatal growth [9]. The contribution of genetic factors to such accelerated postnatal growth in those born SGA is unknown. We hypothesized that certain obesogenic gene variants would have a disproportionately higher impact in increasing early childhood BMI in those born SGA than those born appropriate for gestational age (AGA), and therefore explain part of the link between low birthweight and subsequent risk of obesity. This is based on the assumption that underlying metabolic programming in those born SGA would enhance the biological metabolic effect of any Mendelian inherited gene variant. We explored the childhood growth of those born SGA and AGA and investigated the association of candidate obesity genes with the BMI z-score in the Auckland Birthweight Collaborative (ABC) study children, in relation to SGA status.

\section{Methods}

The Auckland birthweight collaborative study

The ABC Study has been described in more detail elsewhere [10]. The study principally investigates factors that relate to childhood growth and development. All children included in this study were born at 37 weeks of gestation or more. Infants were defined as SGA if their birthweight was equal to or below the sex-specific 10th percentile for gestational age in the New Zealand population. Infants were defined as AGA if their birthweight was greater than the sex-specific 10th percentile for gestational age in the New Zealand population.

\section{Subjects}

Eligible for inclusion in the ABC study were all SGA infants and a selection of one in sixteen AGA infants, born in the Waitemata Health or Auckland Healthcare regions in New Zealand between 16 October 1995 and 12 August 1996. All SGA children and a selection of one in eight AGA children, who were born between 12 August 1996 and 30 November 1997 in the Auckland Healthcare region, were also eligible for inclusion. Data have been collected at birth (phase 1), 12 months (phase 2), 3.5 years (phase 3), 7 years (phase 4), and 11 years (phase 5). This study utilises phases 3,4 , and 5 data. A total of 547 children (228 SGA and 319 AGA, phase 5) have completed BMI and 542 children have genotype data. The sample was restricted to children who were born to European mothers because of the poor response rate from non-European ethnic groups at earlier phases. DNA was extracted from the blood/buccal samples using Qiagen's DNA extraction kit and following the manufacturer's instructions. The study received ethical approval from the Northern Regional Ethics Committee. The 3.5 year old children's study had the ethic approval number of $99 / 097$; the study of 7 year old children is $\mathrm{AKX} / 02 / 00 / 319$; and the study of 11 year old children is NYT/06/00/112. Signed consent for the study was given by the parents of the children at all phases and also given by the child at age 11 years.

\section{Outcome measure}

Body Mass Index (weight/height ${ }^{2}$ ) is a common method of assessing obesity status that was used in this study. BMI scores were converted to z-scores using the LMS method to ensure normal distribution of the data [11].

\section{SNP selection}

The SNPs were selected from a systematic literature search to identify genetic variants demonstrating association with obesity using the criteria previously described [12]. We included 37 obesity SNPs identified from published candidate gene or genome-wide association studies, located in 21 genes. Genotypic data were not available for nine SNPs: rs1387153 (MTNR1B), rs6020339 (CTNNBL1), rs2388399 (PFKP), rs477181 (MC4R), rs3820152 (ADIPOR1), rs7561317 (TMEM18), rs7647305 (ETV5 - DGKG), rs2844479 (NCR3 - AIF1), and rs10487818 (NAMPT). These SNPs either could not be multiplexed into our sequenom assays, failed or did not pass our quality control measure for inclusion in the analysis. Table 1 provides the list of genes/SNPs selected for investigation including the tested allele and its frequency.

\section{Genotyping}

Genotyping was performed with the MassARRAY and iPlex systems of the Sequenom genotyping platform (Sequenom, San Diego, CA), which uses the MALDITOF primer extension assay $[13,14]$, according to manufacturer's recommendations. Assays were optimized in 24 samples consisting of 20 reference Centre d'Etude du Polymorphisme Humain (CEPH) samples and 4 blanks. All sample plates contained cases, controls, blanks, $\mathrm{CEPH}$ and duplicate samples. Quality control measures included independent double genotyping, blind to sample identity and blind to the other caller, and where available, comparison of our CEPH genotypes to those in the HapMap (www.hapmap.org).

\section{Statistical analysis}

Generalised linear model was used to test the linearity of genotype-phenotype relationship for quantitative traits. Since the outcomes of interest, BMI z-score, was a repeated measure, the GENMOD procedure with the REPEATED statement in SAS was used to fit a generalised estimating equations (GEE) model. For linearity of the genotype-phenotype relationship for quantitative 
Table 1 Obesity SNPs for association with BMI in the Auckland Birthweight Collaborative study samples (Morgan et al. [12])

\begin{tabular}{|c|c|c|c|c|c|c|}
\hline Gene & Name & Chr & SNP & Description & $\begin{array}{l}\text { Tested } \\
\text { allele }\end{array}$ & $\begin{array}{l}\text { Tested allele } \\
\text { frequency }\end{array}$ \\
\hline ADIPOR2 & adiponectin receptor 2 & 12 & rs2286385 & Intronic & $\mathrm{T}$ & 0.339 \\
\hline BDNF & Brain-derived neurotrophic factor & 11 & rs6265 & Missense V66M & G & 0.828 \\
\hline BDNF & Brain-derived neurotrophic factor & 11 & rs925946 & $9.2 \mathrm{~kb}$ upstream & G & 0.653 \\
\hline CTNNBL1 & catenin (cadherin-associated protein), beta-like 1 & 20 & rs16986921 & Intronic & $\mathrm{T}$ & 0.046 \\
\hline CTNNBL1 & catenin (cadherin-associated protein), beta-like 1 & 20 & rs6013029 & Intronic & $\mathrm{T}$ & 0.045 \\
\hline CTNNBL1 & catenin (cadherin-associated protein), beta-like 1 & 20 & rs6020395 & Intronic & C & 0.049 \\
\hline CTNNBL1 & catenin (cadherin-associated protein), beta-like 1 & 20 & rs6020712 & Intronic & A & 0.046 \\
\hline CTNNBL1 & catenin (cadherin-associated protein), beta-like 1 & 20 & rs6020846 & Intronic & G & 0.054 \\
\hline CTNNBL1 & catenin (cadherin-associated protein), beta-like 1 & 20 & rs6096781 & Intronic & C & 0.040 \\
\hline CTNNBL1 & catenin (cadherin-associated protein), beta-like 1 & 20 & rs6125962 & Intronic & C & 0.053 \\
\hline FAIM2 & Fas apoptotic inhibitory molecule 2 & 12 & rs7138803 & Intergenic & A & 0.373 \\
\hline FTO & fat mass and obesity associated gene & 16 & rs6499640 & Intronic & A & 0.613 \\
\hline FTO & fat mass and obesity associated gene & 16 & rs8050136 & Intronic & A & 0.358 \\
\hline FTO & fat mass and obesity associated gene & 16 & rs9939609 & Intronic & A & 0.346 \\
\hline GNPDA2 & glucosamine-6-phosphate deaminase 2 & 4 & rs10938397 & $\begin{array}{l}453.9 \mathrm{~kb} \\
\text { downstream }\end{array}$ & G & 0.462 \\
\hline HMGA2 & high mobility group AT-hook 2 & 12 & rs1042725 & UTR-3 & C & 0.502 \\
\hline INSIG2 & insulin-induced gene 2 gene & 2 & rs2012693 & $25.8 \mathrm{~kb}$ upstream & C & 0.607 \\
\hline INSIG2 & insulin-induced gene 2 gene & 2 & rs7566605 & $10 \mathrm{~kb}$ upstream & G & 0.678 \\
\hline KCTD15 & potassium channel tetramerization domain containing 15 & 19 & rs11084753 & 17 kb downstream & A & 0.347 \\
\hline LPL & lipoprotein lipase & 8 & rs3200218 & 3NTR & G & 0.238 \\
\hline MAF & $\begin{array}{l}\text { v-maf musculoaponeurotic fibrosarcoma oncogene } \\
\text { homolog }\end{array}$ & 16 & rs1424233 & $48 \mathrm{~kb}$ downstream & A & 0.479 \\
\hline MC4R & melanocortin-4 receptor & 18 & rs12970134 & 153.8 kb upstream & A & 0.259 \\
\hline MC4R & melanocortin-4 receptor & 18 & rs17700633 & 109.1 kb upstream & A & 0.309 \\
\hline MC4R & melanocortin-4 receptor & 18 & rs17782313 & 187.5 kb upstream & C & 0.228 \\
\hline MC4R & melanocortin-4 receptor & 18 & rs4450508 & 125.1 kb upstream & A & 0.325 \\
\hline MC4R & melanocortin-4 receptor & 18 & rs502933 & $142 \mathrm{~kb}$ upstream & A & 0.317 \\
\hline $\mathrm{MTCH} 2$ & mitochondrial carrier homolog 2 & 11 & rs10838738 & Intronic & G & 0.363 \\
\hline MTMR9 & myotubularin-related protein 9 & 8 & rs2293855 & intronic & A & 0.393 \\
\hline NPC1 & Niemann-Pick disease, type C1 & 16 & rs1805081 & Missense H 215R & A & 0.558 \\
\hline NEGR1 & Neuronal growth regulator 1 & 1 & rs2568958 & $16.7 \mathrm{~kb}$ downstream & A & 0.609 \\
\hline NEGR1 & Neuronal growth regulator 1 & 1 & rs2815752 & 64 kb downstream & $\mathrm{T}$ & 0.608 \\
\hline PFKP & platelet type phosphofructokinase & 10 & rs2388395 & $317 \mathrm{~kb}$ upstream & G & 0.988 \\
\hline PFKP & platelet type phosphofructokinase & 10 & rs6602024 & Intronic & A & 0.106 \\
\hline PTER & phosphotriesterase-related gene & 10 & rs10508503 & $179 \mathrm{~kb}$ upstream & C & 0.917 \\
\hline SEC16B & SEC16 homolog B & 1 & rs10913469 & Intronic & C & 0.172 \\
\hline $\mathrm{SH} 2 \mathrm{~B} 1$ & SH2B adaptor protein 1 & 16 & rs7498665 & Missense A434T & G & 0.614 \\
\hline TMEM18 & transmembrane protein 18 & 2 & rs6548238 & $33 \mathrm{~kb}$ upstream & C & 0.833 \\
\hline
\end{tabular}


Table 2 Analysis of association between SNPs and repeated measures of BMI z-score

\begin{tabular}{|c|c|c|c|c|c|c|c|c|c|c|c|c|c|c|}
\hline \multirow[t]{2}{*}{ Gene } & \multirow[t]{2}{*}{ SNP } & \multirow[t]{2}{*}{$\begin{array}{l}\text { Tested } \\
\text { allele }\end{array}$} & \multicolumn{3}{|c|}{ AGA } & \multicolumn{3}{|l|}{ SGA } & \multicolumn{3}{|c|}{$\begin{array}{l}\text { Adjusted for } \\
\text { SGA status }\end{array}$} & \multicolumn{3}{|c|}{$\begin{array}{c}\text { Interaction with SGA } \\
\text { status }(\text { ref }=A G A)\end{array}$} \\
\hline & & & $\begin{array}{l}\text { Estimate } \\
(95 \% \mathrm{Cl})\end{array}$ & $\begin{array}{c}\mathrm{p} \\
\text { value }\end{array}$ & $\begin{array}{c}\mathrm{q} \\
\text { value }\end{array}$ & $\begin{array}{l}\text { Estimate } \\
(95 \% \mathrm{Cl})\end{array}$ & $\begin{array}{c}p \\
\text { value }\end{array}$ & $\begin{array}{c}q \\
\text { value }\end{array}$ & $\begin{array}{l}\text { Estimate } \\
(95 \% \mathrm{Cl})\end{array}$ & $\begin{array}{c}p \\
\text { value }\end{array}$ & $\begin{array}{c}q \\
\text { value }\end{array}$ & $\begin{array}{l}\text { Estimate } \\
(95 \% \mathrm{Cl})\end{array}$ & $\begin{array}{c}p \\
\text { value }\end{array}$ & $\begin{array}{c}q \\
\text { value }\end{array}$ \\
\hline IPOR2 & 2286385 & $T$ & $0.165(0.065-0.265)$ & 0.0012 & $0.0073^{*}$ & $0.035(-0.093-0.162)$ & 0.5950 & 0.3941 & $10(0.031-0.190)$ & 0.0063 & $0.0207^{*}$ & $-0.131(-0.294-0.031)$ & 0.1134 & 0.1176 \\
\hline BDNF & rs6265 & G & $0.123(0.002-0.244)$ & 0.0470 & 0.0650 & $203(0.040-0.365)$ & 0.0146 & $0.0303^{*}$ & $159(0.059-0.260)$ & 0.0018 & $0.0095^{*}$ & $0.079(-0.123-0.282)$ & 0.4429 & 0.3117 \\
\hline NNE & rs16986 & T & $0.231(-0.023-0.484)$ & 00742 & 848 & $259(-0.058-0.576)$ & 39 & 16 & $042-0449)$ & 80 & * & $-0.377-0.436)$ & 67 & 0.4 \\
\hline NNBL1 & rs6013029 & T & 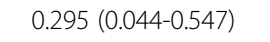 & 0.0214 & $0.0368^{*}$ & $0.310(-0.015-0.634)$ & 0.0611 & 0.0707 & $0.303(0.099-0.507)$ & 0.0036 & $0.0149 *$ & $0.016(-0.396-0.428)$ & 0.9401 & 0.5099 \\
\hline CTNNBL1 & rs6020395 & C & $0.185(-0.043-0.413)$ & 0.1108 & 1164 & $458(0.126-0.791)$ & 0.0069 & $0.0212^{*}$ & $0.303(0.109-0.498)$ & 0.0022 & $0.0108^{*}$ & $0.274(-0.130-0.677)$ & 0.1836 & 0.1608 \\
\hline CTNNBL1 & rs6020712 & A & $0.309(0.050-0.568)$ & 0.0193 & $0.0357^{*}$ & $0.233(-0.074-0.539)$ & 0.1372 & 0.1352 & $0.270(0.068-0.472)$ & 0.0087 & 0.0227 & $-0.077(-0.479-0.325)$ & 0.7085 & 0.4432 \\
\hline NNBL1 & rs 602 & G & 446) & 0.0591 & - & $0.287(-0.0$ & 0.0663 & - & 38) & 085 & - & 52) & 0.7265 & 0.4509 \\
\hline 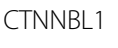 & r & C & 0 & 0.0805 & 0.0894 & ) & 0 & 0.1370 & $0.249(0.034-0.464)$ & 0.0232 & 0.0389 & b) & 2 & 0 \\
\hline NN & 962 & C & 0.20 & 00 & 0.0811 & 2) & 3 & 0.0859 & $0.237(0.054-0.421)$ & 3 & 0. & 0.6 & 6 & 0. \\
\hline FAIM2 & rs7138803 & A & $0.117(0.015-0.219)$ & 0.0249 & $0.0407^{*}$ & $-0.015(-0.150-0.119)$ & 0.8220 & 0.4799 & $0.059(-0.023-0.141)$ & 0.1609 & 0.1492 & $-0.132(-0.301-0.037)$ & 0.1250 & 0.1279 \\
\hline FTO & rs6499640 & A & $0.051(-0.043-0.145)$ & 0.2905 & 0.2290 & $0.153(0.046-0.260)$ & 0.0050 & $0.0188^{*}$ & $0.097(0.027-0.168)$ & 0.0070 & $0.0212^{*}$ & $0.103(-0.039-0.246)$ & 0.1562 & 0.1466 \\
\hline FTO & rs8050136 & A & 0.143 & 0.0055 & 0.019 & $-0.263)$ & 0.0335 & 0.048 & $(0.062-0.219)$ & $4.8 \mathrm{e}-04$ & $0.0042^{*}$ & $-0.005(-0.166-0.158)$ & 0.9569 & 0.5130 \\
\hline FTO & rs9939609 & A & $0.148(0.048-0.248)$ & 0.0038 & $0.0150^{*}$ & $0.154(0.028-0.279)$ & 4 & 0 & $0.300(0.117-0.482)$ & 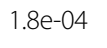 & c & $0.007(-0.154-0.169)$ & 0.9290 & 0.5099 \\
\hline HMGA2 & rs1042725 & C & $-0.198)$ & 0.0304 & $0.0470^{*}$ & $0.062(-0.058-0.183)$ & 0.3 & 0.2411 & $0.087(0.013-0.161)$ & 0.0215 & $0.0368^{*}$ & $-0.041(-0.194-0.112)$ & 0.6015 & 0.3951 \\
\hline KCTD15 & rs11084753 & A & $0.067(-0.036-0.169)$ & 0.2019 & 0.1749 & $0.155(0.037-0.274)$ & 0.0104 & $0.0234^{*}$ & $0.103(0.026-0.181)$ & 0.0090 & $0.0227^{*}$ & $0.088(-0.069-0.244)$ & 0.2732 & 0.2199 \\
\hline MAF & rs 1424233 & A & $0.052(-0.042-0.146)$ & 0.2758 & 0.2199 & $-0.130(-0.263-0.003)$ & 0.0552 & 0.0725 & $-0.024(-0.103-0.054)$ & 0.5446 & 0.3669 & $-0.183(-0.347--0.020)$ & 0.0280 & $0.0441^{*}$ \\
\hline $4 R$ & 134 & A & ( & $1.0 \mathrm{e}-08$ & $-07 *$ & $-0.060(-0.204-0.085)$ & 0.4194 & 0.30 & (10.0 & 4 & 0.0 & $-0.350(-0.525--0.174)$ & 05 & 0.00 \\
\hline C4R & rs & $n$ & 0 & S & 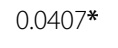 & $-0.047(-0.164-0.070)$ & 0 & 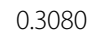 & $0.044(-0$ & S & 99 & $-0.167(-0.325--0$ & 74 & 0.0 \\
\hline MC4R & rs17782313 & C & $0.204(0.105-0.303)$ & $5.6 \mathrm{e}-05$ & $0.0011^{*}$ & $-0.006(-0.147-0.136)$ & 0.9398 & 0.5099 & $0.122(0.041-0.204)$ & 0.0032 & $0.0140^{*}$ & $-0.210(-0.383--0.037)$ & 0.0176 & $0.0346^{*}$ \\
\hline MC4R & rs4450508 & A & $0.189(0.091-0.286)$ & $1.5 \mathrm{e}-04$ & $0.0019^{*}$ & $0.021(-0.115-0.158)$ & 0.7610 & 0.4622 & $0.122(0.041-0.202)$ & 0.0029 & $0.0134^{*}$ & $-0.168(-0.336-0.0)$ & 0.0496 & 0.0674 \\
\hline MC4R & rs502933 & A & $0.168(0.070-0.266)$ & $7.5 \mathrm{e}-04$ & $0.0053^{*}$ & $0.009(-0.129-0.148)$ & 0.8944 & 0.4999 & $0.107(0.027-0.188)$ & 0.0092 & $0.0227^{*}$ & $-0.159(-0.329-0.011)$ & 0.0665 & 0.0794 \\
\hline MTMR9 & rs2293855 & A & $0.102(0.0020 .801)$ & 0.0013 & $0.0073^{*}$ & $-0.039(-0.156-0.079)$ & 0.5208 & - & $0.079(0.003-0.156)$ & 0.0427 & 0.0601 & $-0.199(-0.353--0.045)$ & 0.0113 & $0.0241 *$ \\
\hline NEGP & rs 256 & A & $-0.053(-0.139-0.033)$ & 2280 & 1053 & $0.135(0.021-0.250)$ & 0.0203 & 0.036 & $0.021(0.0$ & 0.5526 & 1 & 2) & 097 & 0.022 \\
\hline NEGR1 & rs2815752 & T & $-0.059(-0.146-0.028)$ & 0.1810 & 0.1603 & $0.133(0.021-0.245)$ & 0.0195 & $0.0357^{*}$ & $0.017(0.052-0.085)$ & 0.6359 & 0.4108 & $0.193(0.052-0.334)$ & 0.0075 & $0.0214^{*}$ \\
\hline PTER & rs10508503 & C & $0.307(0.166-0.448)$ & $2.1 \mathrm{e}-05$ & $8.2 \mathrm{e}-04^{*}$ & $-0.009(-0.258-0.240)$ & 0.9446 & 0.5099 & $0.190(0.055-0.324)$ & 0.0057 & $0.0195^{*}$ & $-0.314(-0.602--0.026)$ & 0.0327 & $0.0489 *$ \\
\hline SEC16B & rs10913469 & C & $0.215(0.089-0.341)$ & $8.4 \mathrm{e}-04$ & $0.0053^{*}$ & $99(0.048-0.350)$ & 0.0098 & $0.0227^{*}$ & $0.207(0.110-0.305)$ & $3.1 \mathrm{e}-05$ & $8.2 e-04^{*}$ & $-0.016(-0.213-0.181)$ & 0.8759 & 0.4966 \\
\hline $\mathrm{SH} 2 \mathrm{~B} 1$ & rs7498665 & G & $(-0.191--0.008)$ & 334 & $0.0489^{*}$ & (0.043-0.283) & 0.0076 & $0.0214^{*}$ & $0.080)$ & 0.8664 & 0.4966 & 4) & $6.3 e-04$ & $0.0050^{*}$ \\
\hline
\end{tabular}

* Remain significant after applying multiple testing correction using false discovery rate. 
Table 3 Differences in birth weight and BMI z-score

\begin{tabular}{|c|c|c|c|c|c|}
\hline & & \multicolumn{2}{|c|}{ Birth weight (g) } & \multicolumn{2}{|c|}{ BMI z-score } \\
\hline & & Estimate $(95 \% \mathrm{Cl})$ & $p$ & Estimate $(95 \% \mathrm{Cl})$ & $p$ \\
\hline \multirow[t]{4}{*}{ At Birth } & Female & $-146.0(-220.7--71.2)$ & $1.35 \mathrm{e}-04$ & & \\
\hline & Male & 0 & & & \\
\hline & SGA & $-896.3(-959.4--833.1)$ & $<1 \mathrm{e}-29$ & & \\
\hline & AGA & 0 & & & \\
\hline \multirow[t]{4}{*}{3.5 year old } & Female & & & $-0.18(-0.32--0.05)$ & 0.0075 \\
\hline & Male & & & 0 & \\
\hline & SGA & & & $-0.46(-0.60--0.33)$ & $6.26 \mathrm{e}-11$ \\
\hline & AGA & & & 0 & \\
\hline \multirow[t]{4}{*}{7 year old } & Female & & & $-0.15(-0.33-0.03)$ & 0.0935 \\
\hline & Male & & & 0 & \\
\hline & SGA & & & $-0.38(-0.56--0.20)$ & $3.88 \mathrm{e}-05$ \\
\hline & AGA & & & 0 & \\
\hline \multirow[t]{4}{*}{11 year old } & Female & & & $-0.29(-0.47--0.11)$ & 0.0017 \\
\hline & Male & & & 0 & \\
\hline & SGA & & & $-0.26(-0.44-0.08)$ & 0.0058 \\
\hline & AGA & & & 0 & \\
\hline
\end{tabular}

traits, additive model was used, each SNP was coded 0, 1 , and 2 for each tested allele [15]. The association with SNPs was carried out in four ways due to the disproportionate sampling of AGA and SGA at birth (phase 1): using stratified by (1) AGA and (2) SGA children, (3) using a main effect model with adjustment for the SGA status, and (4) interaction with SGA status. False discovery rate (FDR) q-values were calculated for the total number of tests (148 tests; 37 SNPs times four analyses) using the 'qvalue' package in $\mathrm{R}[16,17]$ with the null hypothesis fraction estimated by the smoothing method of Storey and Tibshirani [18]. A prespecified FDR threshold was set at 0.05 . Table 2 provides the results of the analysis of association between SNPs and BMI z-score with both q-values and unadjusted p-values; a SNP is called significant if the q-value is less than the prespecified 0.05. SAS (V9.2 SAS Institute., Cary, NC, USA) and R (R: A language and environment for statistical computing. $\mathrm{R}$ Foundation for Statistical Computing, Vienna, Austria. ISBN 3-900051-07-0, http://www.R-project.org) were used for statistical analyses. This resulted in a pvalue of 0.05 as being statistically significant.

\section{Results}

\section{Growth patterns of $A B C$ participants according to SGA} status

SGA children had significantly lower BMI z-score than AGA children at all three assessments conducted at 3.5, 7 and 11 years of age (Table 3 and Figure 1). BMI z-score showed no significant interaction between SGA status and age at assessment $(\mathrm{p}=0.254)$ (Table 4 and Figure 2 ).

\section{Genotype data}

A total of 37 candidate SNPs for obesity were successfully genotyped (Table 1), and their relationship with BMI z-score was tested. There was no indication of a deviation from Hardy Weinberg equilibrium (HWE), except for one SNP (rs7903146) which was excluded from further analysis. Using a prespecified FDR threshold at $\alpha=0.05$ [17], we determined that 27 SNPs were significantly associated with the BMI z-score in at least one of

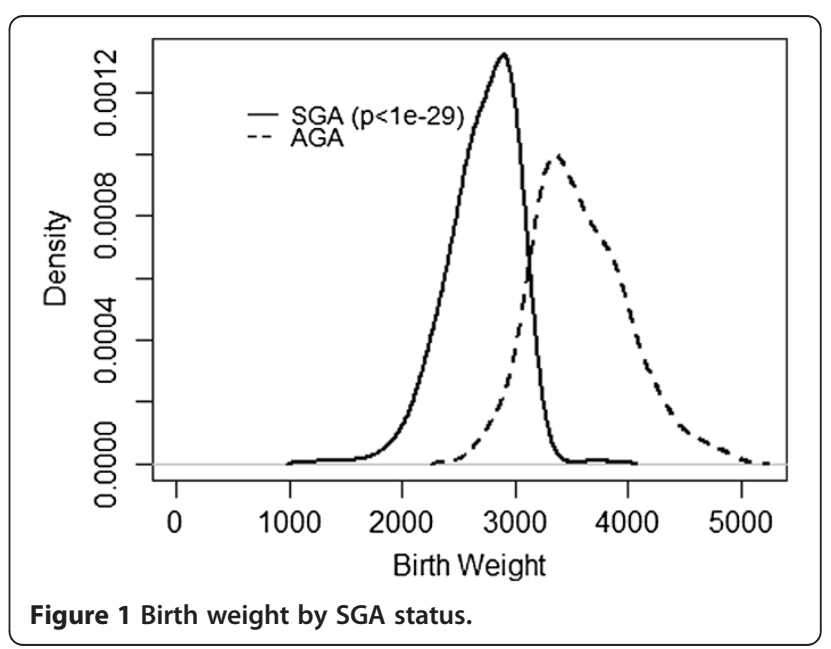


Table 4 BMI z-score Growth by SGA status

\begin{tabular}{|c|c|c|c|c|c|c|c|}
\hline \multirow[b]{2}{*}{ Phase } & \multicolumn{2}{|c|}{ AGA } & \multicolumn{2}{|c|}{ SGA } & \multicolumn{2}{|c|}{ Adjustment for SGA status } & \multirow{2}{*}{$\frac{\text { Interaction with SGA statu }}{p}$} \\
\hline & Estimate (SE) & $p$ & Estimate (SE) & $p$ & Estimate (SE) & $p$ & \\
\hline At 3.5 year & 0 & & 0 & & 0 & & 0.254 \\
\hline At 7 year & $0.17(0.08)$ & 0.0401 & $0.25(0.11)$ & 0.0178 & $0.20(0.06)$ & 0.0018 & \\
\hline At 11 year & $0.14(0.08)$ & 0.087 & $0.34(0.10)$ & 0.0008 & $0.22(0.06)$ & $4.03 e-04$ & \\
\hline
\end{tabular}

the four analyses: stratified by AGA and SGA children, after adjustment for SGA status, and interaction with SGA status (Table 2).

Variants associated with BMI z-score in AGA children A total of 16 variants: rs2286385 (ADIPOR2), rs6013029 and rs6020712 (CTNNBL1), rs7138803 (FAIM2), rs8050136 and rs9939609 (FTO), rs1042725 (HMGA2), rs12970134, rs17700633, rs17782313, rs4450508, and rs502933 (MC4R), rs2293855 (MTMR9), rs10508503 (PTER), rs10913469 (SEC16B), and rs7498665 (SH2B1) in 10 genes were found to be significantly associated with BMI z-score in AGA children after correction for multiple testing (Table 2). The impact on BMI z-score increase varied with the tested variant allele from 0.100 (rs7498665 SH2B1) to 0.309 (rs6020712 CTNNBL1).

\section{Variants associated with BMI z-score in SGA children}

Ten variants in seven genes were significantly associated with BMI z-score in SGA children after correction for multiple testing (Table 2). The impact on BMI z-score increase varied from 0.133 (rs2815752 NEGR1) to 0.458 (rs6020395 CTNNBL1). In contrast to those born AGA, none of the 5 variants in MC4R were associated with increasing BMI z-score in those born SGA.

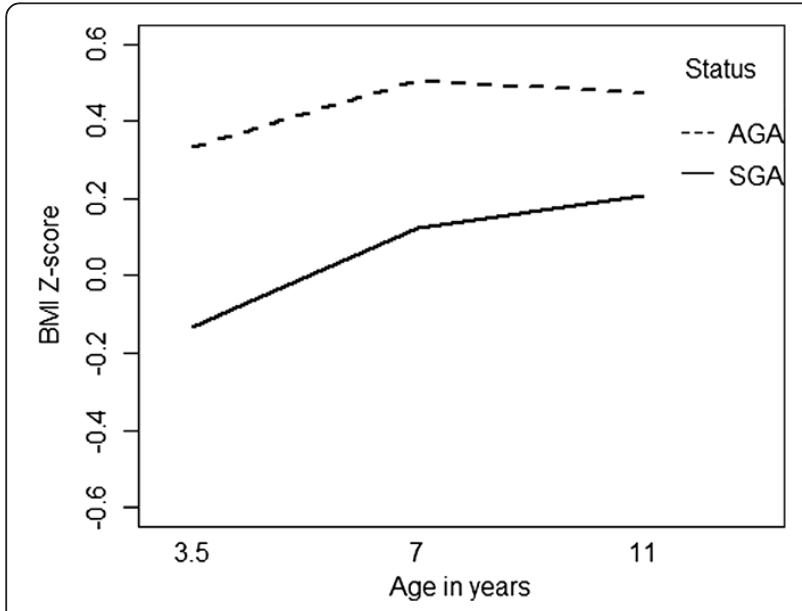

Figure 2 BMI z-score by SGA status.
Variants associated with BMI z-score after adjustment for SGA status

A total of 20 variants: rs2286385 (ADIPOR2), rs6265 (BDNF), rs16986921, rs6013029, rs6020395, rs6020712, rs6020846, rs6096781, and rs6125962 (CTNNBL1), rs6499640, rs8050136, and rs9939609 (FTO), rs1042725 (HMGA2), rs11084753 (KCTD15), rs12970134, rs17782313, rs4450508, and rs502933 (MC4R), rs10508503 (PTER), and rs10913469 (SEC16B) showed significant association after adjustment SGA status and also correction for multiple testing. BMI z-score increased with all tested obesity risk variant alleles ranging from 0.087 (rs1042725 HMGA2) to 0.303 (rs6013029 CTNNBL1).

\section{Variants associated with interaction between BMI z-score} and SGA status

A total of eight variants: rs1424233 (MAF), rs12970134 and rs17782313, (MC4R), rs2293855 (MTMR9), rs2568958 and rs2815752 (NEGR1), rs10508503 (PTER), and rs7498665 (SH2B1) showed significant interaction with SGA status. Two of these variant (MC4R and PTER) also showed a significant association with BMI z-score among the whole cohort with adjustment for SGA status, however, their effect was only observed among those born AGA, with no effect in SGA. Of the remaining obesity risk variants showing a significant interaction with SGA status, two variants in NEGR1 were associated with increased BMI in those born SGA but no effect in those born AGA, while $S H 2 B 1$ obesity risk variant showed increased BMI in those born SGA and reduced BMI in those born AGA. There was a significant interaction between the $M A F$ variant and SGA status, and reduced BMI in the SGA group (Table 2). However, there were no significant effects on BMI when the association was tested within those born SGA and AGA.

\section{Discussion}

We confirmed 27 variants within 14 obesity genes to be individually associated with increasing early childhood BMI in those born AGA, most of which were also associated with increasing early childhood BMI in the whole cohort after adjusting for SGA status. Variants associated with the interaction of BMI z-score and SGA status were largely driven by their predominant association with increasing BMI in either the AGA group $(M C 4 R$, PTER, MTMR9), or the SGA group (NEGR1, SH2B1), 
rather than a consistently larger effect seen in the SGA group as hypothesised. None of the $5 M C 4 R$ obesity risk variants associated with increasing BMI in AGA were significant in those born SGA. However, all three of the FTO obesity risk variants showed an effect of increasing BMI in those born SGA, one of which was also significant in those born AGA.

Although the biological functions of the majority of the obesity gene variants are still being investigated, most of the obesity risk variants are thought to act centrally on increasing hyperphagic drive [19]. The other physiological mechanisms by which these gene variants could be acting to influence postnatal growth would be through a change in control of body's energy partitioning from lean to fat tissue, or shift in metabolic efficiency through thermogenesis.

Our New Zealand SGA birth cohort did not show evidence of accelerated postnatal growth, in terms of their BMI z-score, which remained lower than those born AGA at assessment age 3.5, 7 and 11 years, however, they may still have increased postnatal fat deposition relative to lean body or skeletal mass. There is evidence from studies conducted in Switzerland [20] and Spain [21] that pre-pubertal children born SGA have more body fat and less lean tissue than weight or age matched controls. Nonetheless, the higher cardiovascular risks observed with accelerated postnatal growth in those born SGA have been demonstrated to occur with a rapid tempo of childhood gain in BMI rather than body fat [22].

Our results do not suggest that the known obesity risk gene variants have a disproportionately higher impact on increasing childhood BMI in those born SGA compared to AGA. However, until the age of 11 years, those born SGA remained smaller than AGA children, and it remains to be seen whether they develop a greater prevalence of obesity in adulthood. This work leads us to believe that non-genetic or environmental factors are more likely to be important in influencing childhood BMI in those born SGA.

\section{Conclusions}

In summary, our longitudinal data with measurement of BMI at 3.5, 7 and 11 years of age, confirm that several of the adult obesity risk gene variants $\left(A D I P O R 2, B C D I N 3 D_{-}-\right.$ FAIM2, BDNF, CTNNBL1, FTO, HMGA2 KCTD15, MTMR9 MC4R, PTER, SEC16B, and SH2B1) were also associated with childhood growth in New Zealand. Most of the association between obesity variants with childhood BMI was observed within children born AGA rather than the SGA, suggesting that non-genetic or environmental factors may be more important in influencing childhood BMI in those born SGA.

\section{Abbreviations}

ABC: Auckland Birthweight Collaborative; AGA: Appropriate-for-gestationalage; SGA: Small-for-gestational-age; SNPs: Single nucleotide polymorphisms; CEPH: Centre d'Etude du Polymorphisme Humain; HWE: Hardy Weinberg Equilibrium; MALDI-TOF: Matrix Assisted Laser Desorption/Ionization - Time of Flight.

\section{Competing interests}

The authors declare that they have no competing interests.

\section{Authors' contributions}

DYH performed the data analyses. DYH and RM wrote the manuscript. ARM and WJL performed genotyping and contributed to the writing of the manuscript. LRF, CRW, and KEW contributed to the writing of the manuscript. JMDT had responsibility for outcome assessment and contributed to the writing of the manuscript. EAM supervised the design and execution of the study and contributed to the writing of the manuscript. All authors read and approved the final manuscript.

\section{Acknowledgements}

The initial study was funded by the Health Research Council of New Zealand. The 12 month postal questionnaire was funded by Hawkes Bay Medical Research Foundation. The 3.5 year follow-up study was funded by Child Health Research Foundation, Becroft Foundation and Auckland Medical Research Foundation. The 7 year follow-up study was funded by Child Health Research Foundation. The 11 year follow-up was funded by Child Health Research Foundation and the National Heart Foundation. The genetic component of this study was funded by Child Health Research Foundation. We thank Professors T. Lumley, C.J. Wild and C.M. Triggs from the Department of Statistics, University of Auckland, for statistical consultation. EA Mitchell and JMD Thompson are supported by CureKids. The 7 year follow-up study was conducted in the Children's Research Centre which is supported in part by the Starship Foundation and the Auckland District Health Board. We acknowledge the assistance of Gail Gillies, Barbara Rotherham, and Helen Nagels for contacting or assessing the participants. We sincerely thank the parents and children for participating in this study. DY Han, AR Morgan, WJ Lam, and LR Ferguson are supported by Nutrigenomics New Zealand which is a collaboration between AgResearch Ltd., Plant \& Food Research and The University of Auckland with funding through the Foundation for Research Science and Technology.

\section{Author details}

'Discipline of Nutrition, FM\&HS, The University of Auckland, Auckland, New Zealand. 'Department of Paediatrics, FM\&HS, The University of Auckland, Auckland, New Zealand. ${ }^{3}$ Nutrigenomics New Zealand, Auckland, New Zealand. ${ }^{4}$ Department of Medicine, FM\&HS, The University of Auckland, Auckland, New Zealand.

Received: 12 June 2012 Accepted: 18 January 2013 Published: 22 January 2013

\section{References}

1. Lee PA, Chernausek SD, Hokken-Koelega AC, Czernichow P, International Small for Gestational Age Advisory B: International Small for Gestational Age Advisory Board consensus development conference statement: management of short children born small for gestational age, April 24October 1, 2001. Pediatrics 2003, 111(6 Pt 1):1253-1261.

2. Barker DJ: Maternal nutrition, fetal nutrition, and disease in later life. Nutrition 1997, 13(9):807-813.

3. Bruce KD, Hanson MA: The developmental origins, mechanisms, and implications of metabolic syndrome. J Nutr 2010, 140(3):648-652.

4. Hattersley AT, Tooke JE: The fetal insulin hypothesis: an alternative explanation of the association of low birthweight with diabetes and vascular disease. Lancet 1999, 353(9166):1789-1792.

5. Frayling TM, Timpson NJ, Weedon MN, Zeggini E, Freathy RM, Lindgren CM, Perry JR, Elliott KS, Lango H, Rayner NW, et al: A common variant in the FTO gene is associated with body mass index and predisposes to childhood and adult obesity. Science 2007, 316(5826):889-894.

6. Silventoinen K, Pietilainen KH, Tynelius P, Sorensen TI, Kaprio J, Rasmussen F: Genetic and environmental factors in relative weight from birth to age 18: the Swedish young male twins study. Int J Obes 2007, 31(4):615-621. 
7. Elks CE, Loos RJ, Sharp SJ, Langenberg C, Ring SM, Timpson NJ, Ness AR, Davey Smith G, Dunger DB, Wareham NJ, et al: Genetic markers of adult obesity risk are associated with greater early infancy weight gain and growth. PLoS Med 2010, 7(5):e1000284.

8. Elks CE, Loos RJ, Hardy R, Wills AK, Wong A, Wareham NJ, Kuh D, Ong KK: Adult obesity susceptibility variants are associated with greater childhood weight gain and a faster tempo of growth: the 1946 British Birth Cohort Study. Am J Clin Nutr 2012, 95(5):1150-1156.

9. Ong KK, Loos RJ: Rapid infancy weight gain and subsequent obesity: systematic reviews and hopeful suggestions. Acta Paediatr 2006, 95(8):904-908.

10. Thompson JM, Clark PM, Robinson E, Becroft DM, Pattison NS, Glavish N, Pryor JE, Wild CJ, Rees K, Mitchell EA: Risk factors for small-for-gestationalage babies: The Auckland Birthweight Collaborative Study. J Paediatr Child Health 2001, 37(4):369-375.

11. Cole TJ: The LMS method for constructing normalized growth standards. Eur J Clin Nutr 1990, 44(1):45-60.

12. Morgan AR, Thompson JM, Murphy R, Black PN, Lam WJ, Ferguson LR, Mitchell EA: Obesity and diabetes genes are associated with being born small for gestational age: results from the Auckland Birthweight Collaborative study. BMC Med Genet 2010, 11:125.

13. Jurinke C, van den Boom D, Cantor CR, Koster H: The use of MassARRAY technology for high throughput genotyping. Adv Biochem Eng Biotechnol 2002, 77:57-74.

14. Storm N, Darnhofer-Patel B, van den Boom D, Rodi CP: MALDI-TOF mass spectrometry-based SNP genotyping. Methods Mol Biol 2003, 212:241-262.

15. Balding DJ: A tutorial on statistical methods for population association studies. Nat Rev Genet 2006, 7(10):781-791.

16. Dabney A, Storey JD, Warnes GR: qualue: Q-value estimation for false discovery rate control. $\mathrm{R}$ package version 1.26.0. http://www. bioconductor.org/packages/release/bioc/html/qvalue.html.

17. Storey JD: A direct approch to false discovery rates. J R Stat Soc Ser B (Stat Methodol) 2002, 64(3):479-498.

18. Storey JD, Tibshirani R: Statistical significance for genomewide studies. Proc Natl Acad Sci U S A 2003, 100(16):9440-9445.

19. Choquet H, Meyre D: Genetics of Obesity: What have we Learned? Current genomics 2011, 12(3):169-179.

20. Jornayvaz FR, Selz R, Tappy L, Theintz GE: Metabolism of oral glucose in children born small for gestational age: evidence for an impaired whole body glucose oxidation. Metabolism 2004, 53(7):847-851.

21. Ibanez L, Ong K, Dunger DB, de Zegher F: Early development of adiposity and insulin resistance after catch-up weight gain in small-forgestational-age children. J Clin Endocrinol Metab 2006, 91(6):2153-2158.

22. Krishnaveni GV, Veena SR, Wills AK, Hill JC, Karat SC, Fall CH: Adiposity, insulin resistance and cardiovascular risk factors in 9-10-year-old Indian children: relationships with birth size and postnatal growth. J Dev Orig health Dis 2010, 1(6):403-411.

doi:10.1186/1471-2350-14-10

Cite this article as: Han et al.: Reduced genetic influence on childhood obesity in small for gestational age children. BMC Medical Genetics 2013 14:10.

\section{Submit your next manuscript to BioMed Central and take full advantage of:}

- Convenient online submission

- Thorough peer review

- No space constraints or color figure charges

- Immediate publication on acceptance

- Inclusion in PubMed, CAS, Scopus and Google Scholar

- Research which is freely available for redistribution 